\title{
Trisomy 15 Mosaicism: Challenges in Prenatal Diagnosis
}

\section{Marisa Silva, ${ }^{1 *}$ Cristina Alves, ${ }^{1}$ Sónia Pedro, ${ }^{1}$ Bárbara Marques, ${ }^{1}$ Cristina Ferreira, ${ }^{1}$ José Furtado, ${ }^{1}$ Ana Teresa Martins, ${ }^{2}$ Rosário Fernandes, ${ }^{3}{ }^{3}$ oaquim Correia, ${ }^{2}$ and Hildeberto Correia ${ }^{1}$}

${ }^{1}$ Departamento de Genética Humana, Unidade de Citogenética, Instituto Nacional de Saúde Dr. Ricardo Jorge, INSA, I.P., Lisboa, Portugal

${ }^{2}$ Centro de Diagnóstico Pré-Natal, Maternidade Dr. Alfredo da Costa, Centro Hospitalar de Lisboa Central, Rua Viriato, Lisboa, Portugal

${ }^{3}$ Serviço de Anatomia Patológica, Maternidade Dr. Alfredo da Costa, Centro Hospitalar de Lisboa Central, Rua Viriato, Lisboa, Portugal

Manuscript Received: 23 December 2014; Manuscript Accepted: 11 June 2015

\section{TO THE EDITOR:}

Trisomy 15 mosaicism (mosT15) has been described in fetuses and live-born infants [Christian et al., 1996; Redaelli et al., 2005], with most cases involving confined placental mosaicism (CPM) and meiotic non-disjunction (ND) [EUCROMIC, 1999]. Poor pregnancy outcome prognosis is associated with the presence of aneuploid cells, and there is also a risk of uniparental disomy 15 (UPD15) due to correction of the trisomic state to a disomic constitution. Trisomy or monosomy rescue, gamete complementation and postfertilization error are the main mechanisms leading to UPD and may cause heterodisomy (heteroUPD), isodisomy (isoUPD) or both, depending on the number of meiotic recombinations. The result of maternal (matUPD) and paternal (patUPD) UPD15 is Prader-Willi and Angelman syndrome, respectively, due to imprinting of chromosome region 15q11-15q13. UPD detection can only be achieved using molecular methodologies, such as methylation-specific assays (MSA) [Kotzot, 2008] and, more recently, genome-wide single nucleotide polymorphism (SNP) arrays [Conlin et al., 2010; Schroeder et al., 2013]. MSA allow for methylation pattern analysis of the chromosome regions of interest and SNP-arrays may provide information about copy number as well as UPD, in cases of isoUPD or isodisomy secondary to recombination. HeteroUPD may also be diagnosed by SNParrays if parental and proband DNAs are analyzed in a trio [Conlin et al., 2010; Schroeder et al., 2013]. However, not all molecular methods are equally informative and when a mosaicism is present, especially in a prenatal setting, parent-of-origin analysis as well as karyotype-phenotype correlations become quite challenging. Here we report on a fetus with a CVS diagnosed mosT15 with different degrees of mosaicism found in different tissues and briefly discuss the challenges of prenatal diagnosis of UPD15.

The patient was a 38-year-old, primigravid woman who underwent an in vitro fertilization procedure (IVF) due to infertility after a bilateral salpingectomy. First trimester combined screening at 12 weeks was positive due to increased fetal nuchal translucency $(5.0 \mathrm{~mm}, \geq 95$ th centile). After counseling regarding diagnostic options, fetal karyotyping was offered and the couple opted for CVS which was performed at 12 weeks of gestation. Cleaned villi were (i) finely diced and two MLPA experiments performed (after

\section{How to Cite this Article:}

Silva M, Alves C, Pedro S, Marques B,

Ferreira C, Furtado J, Martins AT,

Fernandes R, Correia J, Correia H. 2015.

Trisomy 15 mosaicism: Challenges in

prenatal diagnosis.

Am J Med Genet Part A 9999:1-4.

DNA extraction by a salting-out in house procedure); and (ii) digested enzymatically with trypsin $\left(0.5 \% 20 \mathrm{~min} ., 37^{\circ} \mathrm{C}, 95 \% \mathrm{RH}\right)$ and collagenase type $\mathrm{V}\left(300 \mathrm{UI} / \mathrm{ml} 2 \mathrm{hr}, 37^{\circ} \mathrm{C}, 95 \% \mathrm{RH}\right)$. An aliquot of the latter was then separated for a MLPA experiment (after DNA extraction using the Intragene Matrix kit, CA) and the remnant was cultured for karyotype analysis using standard methods. MLPA results were consistent with the absence of aneuploidy for chromosomes 13, 18, and 21 in a female fetus, and cytogenetic analysis on long-term CVS cultures revealed the presence of trisomy 15 in 16 of 22 cells examined. In order to evaluate the possibility of a trisomy $15 \mathrm{CPM}$, amniocentesis was offered and performed at 18 weeks of gestation. Chromosome analysis of amniocytes showed a supernumerary chromosome 15 in 14 of 50 metaphases analyzed, thus indicating the presence of a true fetal mosT15. Ultrasound examination at $21+1$ gestational weeks revealed a small ventricular septal defect (VSD), nuchal and pre-nasal edema, hypoplastic

Conflict of interest: None.

Grant sponsor: Portuguese National Health Service.

*Correspondence to:

Marisa Silva, Departamento de Genética Humana, Unidade de Citogenética, Instituto Nacional de Saúde Dr. Ricardo Jorge, INSA, I.P., Avenida Padre Cruz, 1649-016 Lisboa, Portugal.

E-mail: marisa.silva@insa.min-saude.pt

Article first published online in Wiley Online Library

(wileyonlinelibrary.com): 00 Month 2015

DOI 10.1002/ajmg.a.37229 
nasal bone ( $<5$ th centile) and clinodactily of the left hand. Parental karyotypes were normal.

Methylation-specific PCR (MS-PCR) was performed on bisulfate-treated genomic DNA using primers specific for methylated sites within the SNRPN exon 1/promoter region. The sodium bisulfite treatment was done using the Imprint ${ }^{\mathbb{B}}$ DNA Modification Kit (Sigma-Aldrich, Saint Louis, MI) according to manufacturer's instructions. MS-PCR was performed using one common primer that anneals to both alleles and one specific primer each for the methylated (maternal) and unmethylated (paternal) allele as previously described [Zeschnigk et al., 1997]. Amplification products were electrophoresed on an agarose gel along with appropriate positive and negative controls. MS-PCR on the CVS revealed two bands, one for the maternal allele and one for the paternal, the former a little more intense than the latter. Given the presence of the trisomy mosaicism, parent of origin conclusions were not possible. MS-PCR on the AF sample showed the presence of two bands, one for the maternal allele and one for the paternal, but with no discernible difference between them (Fig. 1). After counseling the couple opted for termination of pregnancy which was performed at 22 weeks of gestation. A fetal skin (FS) biopsy was explanted and cultured according to the standard tissue explant technique modified from Verma and Babu [1995] and cytogenetic analysis revealed a low level mosaicism (2\%) for trisomy 15. MSPCR on the FS sample showed similar results to the ones of the AF sample (Fig. 1). Anatomopathological investigations showed a female fetus with $480 \mathrm{~g}, 19 \mathrm{~cm}$ head circumference and $18.4 \mathrm{~cm}$ crown-rump length. External examination revealed hypertelorism, broad flat nasal bridge, micrognathia, low implantation and posterior angulation of the ears and right club foot. The heart examination showed a perimembranous VSD. The placenta was hypoplastic, there was villi edema and a single umbilical artery was present.

SNP array was performed using genomic DNA and the CytoScan HD array (Affymetrix, Santa Clara, CA) was utilized for this analysis and includes more than 2.69 million probes, of which 1.9 million are non-polymorphic copy number probes and 740,000 are SNP probes that genotype with over $99 \%$ accuracy. Genomic

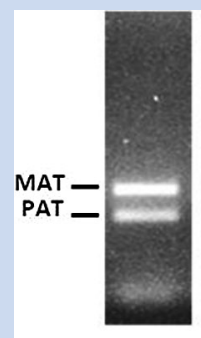

AF
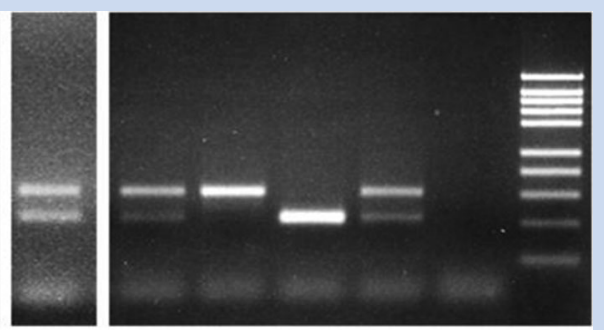

FSB

VCB PWS coordinates are based upon genome build ucsc hg19. Hybridization, data extraction and analysis were done as per manufacturer's protocols. The Affymetrix Chromosome Analysis Suite 2.1.0.16 with NetAffx na33 and Genotype Console v4.0 softwares were used for data analysis and review. SNP array revealed mosaic parental heterodisomy for the entire chromosome 15 (Fig. 2). The mosaic was estimated to be at the level of approximately $29 \%$ and $26 \%$ on the CVS and AF samples repectively and not detected on the FS sample. No monosomy or gains/losses were suggested by the SNP array. Trio analysis was performed using UPDtool as described by Schroeder et al. [2013]. SNP array analysis was performed on CVS, $\mathrm{AF}$, and $\mathrm{FS}$ and on parental blood samples.

To our knowledge, our case shows the fetal mosT15 with the highest level of trisomy 15 on CVS cells, where the mosaicism was not confined to the placenta (as confirmed both on AF and FS) described thus far. This may account for the difficulties in assessing the UPD status as well as the mechanism underlying the mosaic formation. MS-PCR showed biparental contribution and no conclusive indication about UPD although a higher maternal content was suspected. SNP array on the other hand, allowed for the exclusion of copy-number gains/losses, and the absence of large blocks of homozigosity on chromosome 15 suggested a parental heterodisomy, which is consistent with a parental meiosis I ND. Malsegregation in meiosis II, monosomy rescue and somatic events were excluded since they would have resulted in isodisomy 15 . Trio analysis revealed a maternal/paternal ratio of $60 \%$ or $40 \%$, which also suggests a higher maternal contribution and therefore a probable matUD15 (maternal inheritance shown in Table I). We also observed divergent results between karyotype and SNParray analyses regarding trisomic cell levels on CVS (73\% and 29\%, respectively). This type of divergence has been reported before for peripheral blood samples [Conlin et al., 2010] and, in this case, may be due to heterogeneous cell distribution in the placenta and/or cell culture selection, since the villi tissue used for SNP-array was mainly cytotrophoblastic in origin and the cytogenetic analysis was performed on a mixture of cytotrophoblast- and mesenchymeoriginated cultured cells. Nevertheless the confirmation of placental mosaicism on both CVS cell populations also indicates trisomy rescue involvement in the mosaic formation. We assume, therefore, that an initial meiosis I maternal ND occurred, followed by a trisomic conception and a subsequent postzygotic trisomy rescue with the resulting mosaicism and maternal heterodisomy. The advanced maternal age also argues in favor of a maternal ND, since it is a well known risk factor in the etiology of trisomy 15, as well as in other aneuploidies [Ginsburg et al., 2000]. The fact that the pregnancy resulted from an IVF procedure should also be taken into account, as a high frequency of ND has been estimated in IVF patients of advanced reproductive age [Robinson et al., 1999].

UPD investigation is difficult when mosaicism is present, due to the contribution of genetically different cell lines, even when trio analysis is performed [Schroeder et al., 2013]. In our case, the molecular results presented a dilemma in terms of interpretation and counseling. Even though they were not conclusive regarding parent-of-origin, the fetal phenotype described is consistent with previous reports of mosT15, that include congenital heart disease (e.g., VSD, mitral atresia), craniofacial (e.g., hyper-

telorism, broad flat nasal bridge, micrognathia) and skeletal
FIG. 1. MS-PCR analysis from chorionic villi (VCB), amniotic fluid (AF) and fetal skin (FSB) samples and controls. PWS-PWS patient; AS-AS patient; CN-normal control; M-100-1000 bp ladder; Ø-PCR control; MAT-maternal PCR product; PATpaternal PCR product. 


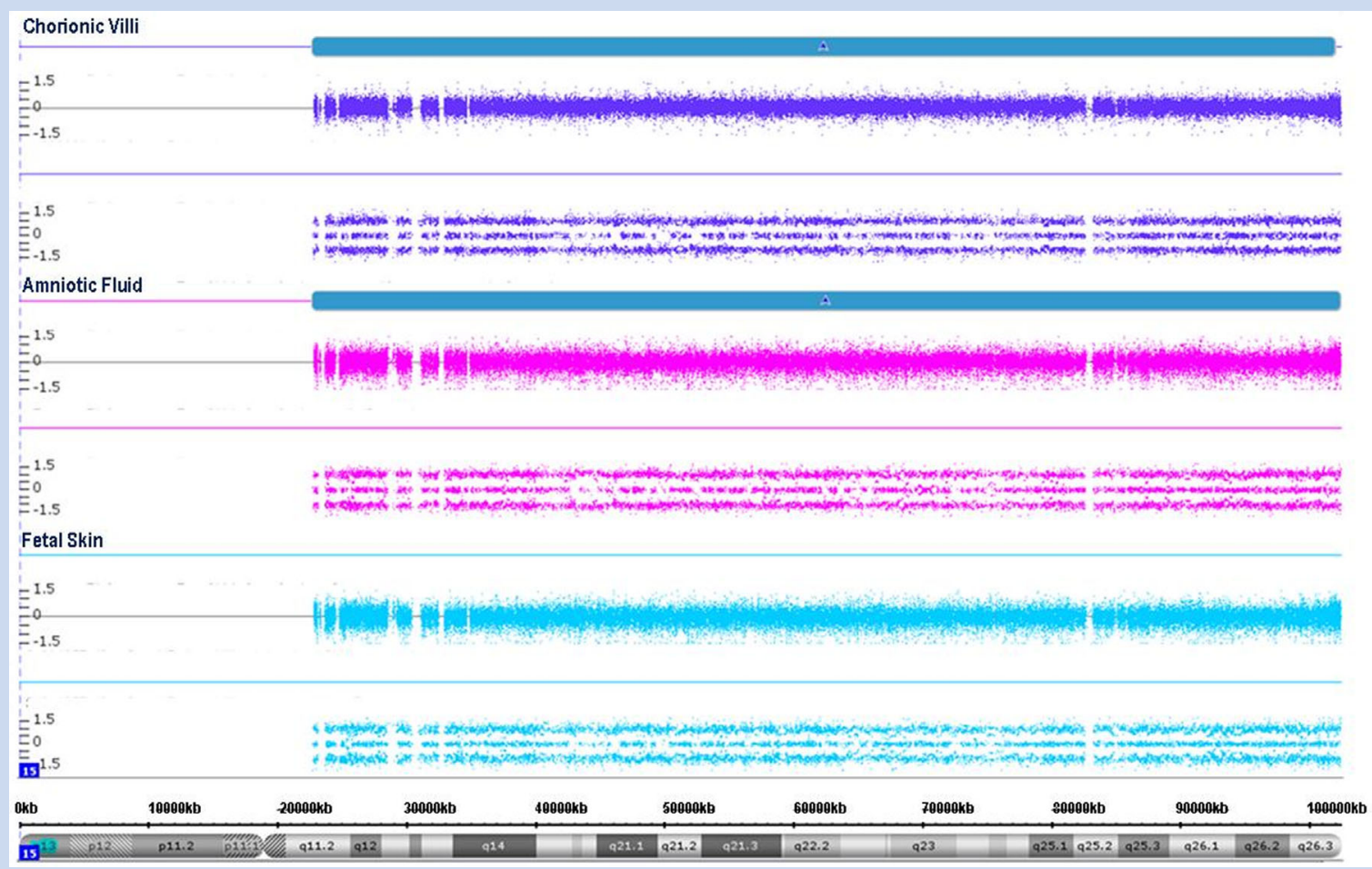

FIG. 2. SNP-array analysis results on the fetal samples showing mosaic gain on CVS and AF samples and no detectable mosaic on the fetal skin sample.

anomalies (e.g., abnormal rib number, clenching/overlapping of the fingers) [Christian et al., 1996; Olander et al., 2000; Redaelli et al., 2005]. PWS was confirmed, through molecular techniques by other authors either due to trisomy 15/matUPD15 mosaicism or non-mosaic matUPD15 and complete maternal heteroUPD15 has been found in around $21-29 \%$ of PWS patients [Tucker et al., 2012] with morphological features consistent with the ones we describe. Nevertheless, given that a confirmed mosT15 was present, with such a high level of abnormal cells on CVS, it is highly likely that the trisomy has had a more negative effect on the phenotype than the presence of UPD itself as previously proposed [Olander et al., 2000]. Even though the fetus we describe was referred at an early gestational age when only increased nuchal translucency was observed, other fetal alterations were found thereafter (both on ultrasound and postmortem), confirming the expected more severe phenotype due to the presence of trisomy 15 . The presence of single umbilical artery as well as of placental abnormalities might lead us to expect IUGR,

TABLE I. Absence of Paternal Contribution to Chromosome 15

$\begin{array}{lc}\text { Probe Set Name } & \text { Chromosomal Position } \\ \text { S-3BDEI } & 26133343 \\ \text { S-3YWBB } & 26406413 \\ \text { S-4BUYR } & 46850579 \\ \text { S-3OMPW } & 56172652 \\ \text { S-3DOIM } & 59941622 \\ \text { S-4EYSM } & 62044136 \\ \text { S-3WATJ } & 70004352 \\ \text { S-4MOCI } & 91676168 \\ \text { S-3VYIN } & 92874777 \\ \text { S-4PMET } & 100366944\end{array}$

$\begin{array}{cc}\text { Father's genotype } & \text { Proband's genotype } \\ A A & B B \\ A A & B B \\ A A & B B \\ B B & A A \\ B B & A A \\ B B & A A \\ A A & B B \\ A A & B B \\ B B & A A \\ B B & A A\end{array}$

Mother's genotype
BB
$A B$
$B B$
$A A$
$A A$
$A A$
$A B$
$B B$
$A B$
$A B$

Shown are examples of informative SNP genotypes from trio analysis using the CytoScan HD SNP microarray (Affymetrix Inc., Santa Clara, CA) and UPDtool (http://www.uni-tuebingen.de/uni/thk/de/fgenomik-software.html). SNP alleles are assigned "A" or "B" at each loci by the Affymetrix Chromosome Analysis software (ChAS; Affymetrix Inc., Santa Clara, CA). The identical genotypes between the proband and mother at the examined loci are consistent with heterodisomy. 
as in previous reports [Christian et al., 1996; Redaelli et al., 2005]. However we did not observed IUGR, possibly due to heterogeneous distribution of the trisomic cell line in the placental tissue that would not render enough severity of transport/metabolic dysfunction to compromise fetal growth.

Differentiating between a mitotic and a meiotic origin for trisomy 15 is, as for other trisomies, important for proper counseling and determining recurrence risks, because trisomy as a result of meiotic ND is associated with a higher risk of recurrence [Christian et al., 1996]. Assessing the mechanism involved is not always a straightforward endeavour in prenatal diagnosis of mosT15 since not all methodologies are equally informative and phenotype information is limited (e.g., there is no possibility of neurological development evaluation). Even in a time when highly accurate and high-throughput DNA-based testing is so widely available, the limitations of those tests should always be taken into consideration, especially before establishing genotype-phenotype correlations.

\section{REFERENCES}

Christian SL, Smith ACM, Macha M, Black SH, Elder FFB, Johnson JM-P, Resta RG, Surti U, Suslak L, Verp MS, Ledbetter DH. 1996. Prenatal diagnosis of uniparental disomy 15 following trisomy 15 mosaicism. Prenat Diagn 16:323-332.

Conlin LK, Thiel BD, Bonneemann CG, Medne L, Ernst LM, Zackai EH, Deardoff MA, Krantz ID, Hakonardson H, Spinner NB. 2010. Mechanisms of mosaicism, chimerism and uniparental disomy identified by single nucleotide polymorphism array analysis. Hum Mol Genet 19:1263-1275.
EUCROMIC. 1999. Trisomy 15 CPM: Probable origins, pregnancy outcome and risk of fetal UPD. Prenat Diagn 19:29-35.

Ginsburg C, Fokstuen S, Shinzel A. 2000. The contribution of uniparental disomy to congenital development defects in children born to mothers at advanced childbearing age. Am J Med Genet 95:454-460.

Kotzot D. 2008. Prenatal testing for uniparental disomy: Indications and clinical relevance. Ultrasound Obstet Gynecol 31:100-105.

Olander E, Stamberg J, Steinberg L, Wulfsberg EA. 2000. Third PraderWilli syndrome phenotype due to maternal uniparental disomy 15 with mosaic trisomy 15. Am J Med Genet 93:215-218.

Redaelli S, Sala E, Roncaglia N, Colombo C, Crosti F, Villa N, Tagliabue P, Cappellini A, Dalprà L. 2005. Severe intrauterine growth restriction and trisomy 15 confined placental mosaicism: A case report and review of the literature. Prenat Diagn 25:140-147.

Robinson WP, Bernasconi F, Lau A, McFadden DE. 1999. Frequency of meiotic trisomy depends on involved chromosome and mode of ascertainment. Am J Med Genet 84:34-42.

Schroeder C, Sturm M, Dufke A, Mau-Holzmann U, Eggermann T, Poths S, Riess O, Bonin M. 2013. UPDtool: A tool for detection of iso- and heterodisomy in parent-child trios using SNP microarrays. Bioinformatics 29:1562-1564.

Tucker T, Schlade-Bartusiak K, Eydoux P, Nelson TN, Brown L. 2012. Uniparental disomy: Can SNP array data be used for diagnosis? Genet Med 14:753-756.

Verma RS and Babu A. 1995. Tissue culture techniques and chromosome preparation. In: Human Chromosomes. Principles and Techniques (Verma RS and Babu A, eds.), McGraw-Hill, New York, 6-71.

Zeschnigk M, Lich C, Buiting K, Doerfler W, Horsthemke B. 1997. A single-tube PCR test for the diagnosis of Angelman and Prader-Willi syndrome based on allelic methylation differences at the SNRPN locus. Eur J Hum Genet 5:94-98. 